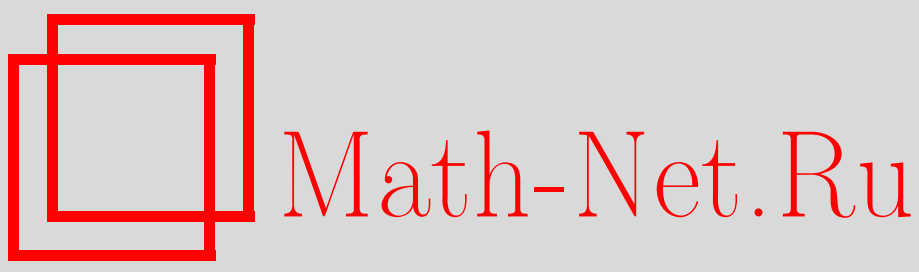

Е. И. Бережной, Оценки равномерного модуля непрерывности функций из симметричных пространств, Изв. РАН. Сер. матем., 1996, том 60, выпуск 2, 3-20

DOI: https://doi.org/10.4213/im69

Использование Общероссийского математического портала Math-Net.Ru подразумевает, что вы прочитали и согласны с пользовательским соглашением

http://www . mathnet.ru/rus/agreement

Параметры загрузки:

IP : 52.6 .47 .48

26 апреля 2023 г., 03:49:30 
УДК 517.5

\author{
Е.И. Бережной
}

\title{
Оценки равномерного модуля непрерывности функций из симметричных пространств
}

\begin{abstract}
Для функции, заданной в $\mathbb{R}^{n}$, доказана многомерная теорема "исправимости" типа теоремы К. Осколкова, а именно, дана точная количественная оценка равномерного модуля непрерывности функции на "больших" множествах, если известна оценка модуля непрерывности этой функции в симметричном пространстве. Показано, что оценка равномерного модуля непрерывности зависит только от фундаментальной функции симметричного пространства.

Библиография: 8 наименований.
\end{abstract}

В настоящей статье дана количественная оценка равномерного модуля непрерывности функции на "больших" множествах, если известна оценка модуля непрерывности этой функции в симметричном пространстве на $\mathbb{R}^{n}$. Полньй ответ на аналогичный вопрос в случае прямой для пространства $L^{p}$ дан $\mathrm{K}$. Осколковым [1]-[3]. Отметим, что некоторые частные результаты в $\mathbb{R}^{n}$ были получены ранее Ю. Брудным [4].

Подход, предлагаемый в настоящей статье, основан на оценках максимальных функций, построенных по специальным семействам множеств, позволил решить сразу многомерную задачу для произвольного симметричного пространства. Оказалось, что оценки равномерного модуля непрерывности зависят только от фундаментальной функции симметричного пространства, в котором вычисляется исходный модуль непрерывности, и одинаковы для всех симметричных пространств с данной фундаментальной функцией. Основной результат работы представлен в теореме 1. Отметим, что в данной работе приведен результат (теорема 4), который показывает, что теорема 1 не может быть усилена даже в случае $n=1$. Таким образом, даже в одномерном случае основной результат статьи является содержательным обобшением теоремы К. Осколкова на случай симметричных пространств.

Пусть $D$ - измеримое множество. Через $\chi(D)$ обозначим его характеристическую функцию, а через $m(D)$ его меру Лебега. Пусть $Q-$ единичный куб в $\mathbb{R}^{n}$ с обычной мерой Лебега, $S(m)$ - множество измеримых функций на $Q$. Для $f: Q \rightarrow \mathbb{R}$, как обычно, через $\lambda(f, \alpha)$ обозначим функцию распределения функции $f$, т. е.

$$
\lambda(f, \alpha)=m(\{t:|f(t)|>\alpha\}), \quad \alpha \in \mathbb{R}_{+}
$$

Напомним [5], что банахово пространство функций называется симметричнылм, если из справедливости при всех $\alpha \in \mathbb{R}_{+}$неравенства $\lambda(f, \alpha) \leqslant \lambda(g, \alpha)$ и $g \in X$ следует, что $f \in X$ и $\|f|X\|\leqslant\| g| X\|$ (символом $\|f \mid X\|$ обозначается норма элемента $f$ в пространстве $X)$. Если $X$ - симметричное пространство, то через $X^{\prime}$

(C) Е.И. БЕРЕЖной 1996 
обозначается пространство интегральных функционалов на $X$ с обычной нормой

$$
\left\|g \mid X^{\prime}\right\|=\sup \left\{\int_{Q} g(x) f(x) d x:\|f \mid X\| \leqslant 1\right\} .
$$

Пространство $X^{\prime}$ также является симметричным.

Важную информацию о симметричном пространстве $X$ несет его фундаментальная функция, которая определяется равенством $\psi(X, t)=\|\chi(D) \mid X\|$, где $t=m(D)$. Для фундаментальных функций $X$ и $X^{\prime}$ известно [5] соотношение

$$
\psi(X, t) \psi\left(X^{\prime}, t\right)=t
$$

Напомним [5], что симметричное пространство $X$ называется правильны.м, если для любой $f \in X$ выполнено соотношение

$$
\lim _{m(D) \rightarrow 0}\|\chi(D) f \mid X\|=0
$$

Через $\bar{U}$ обозначим множество квазивогнутых функций $\varphi:[0,1] \rightarrow \mathbb{R}_{+}$, т.е. функций $\varphi$, которые не убывают и для которых отношение $t^{-1} \varphi(t)$ не возрастает. Через $U$ обозначим подмножество в $\bar{U}$, состояшее из функций, которые удовлетворяют дополнительному условию $\lim _{t \rightarrow 0} \varphi(t)=0$. Хорошо известно, что каждая квазивогнутая функция эквивалентна своей наименьшей вогнутой мажоранте.

Отметим, что для каждого симметричного пространства его фундаментальная функция $\psi(X, t)$ квазивогнута, если же $X$ - правильное симметричное пространство, то его фундаментальная функция принадлежит множеству $U$.

Ниже мы будем рассматривать только симметричные пространства, обладаюшие свойством Фату, т.е. те пространства, единичный шар которых замкнут относительно сходимости по мере, или, что эквивалентно, для которых верно равенство $X^{\prime \prime}=X$.

Важными примерами симметричных пространств, обладаюших свойством $\Phi а-$ ту, являются пространства Лебега $L^{p}(p \geqslant 1)$, пространства Орлича $L_{h}$, построенные по выпуклой на $\mathbb{R}_{+}$функции $h(t)$, удовлетворяющей условиям

$$
\lim _{t \rightarrow 0} t^{-1} h(t)=0, \quad \lim _{t \rightarrow \infty} t^{-1} h(t)=\infty
$$

с нормой, которая задается равенством

$$
\left\|f \mid L_{h}\right\|=\inf \left\{\lambda>0: \int_{Q} h\left(\frac{|f(x)|}{\lambda}\right) d x \leqslant 1\right\}
$$

и пространства Лоренща $\Lambda(\varphi)$, Марцинкевича $M(\varphi)$, построенные по $\varphi \in U$, норма в которых задается равенствами

$$
\left\|f\left|\Lambda(\varphi)\left\|=\int_{0}^{\infty} \varphi(\lambda(f, \alpha)) d \alpha, \quad\right\| f\right| M(\varphi)\right\|=\sup _{D} \frac{\varphi(m(D))}{m(D)} \int_{D}|f(x)| d x .
$$

Для фундаментальных функций этих пространств справедливы равенства

$$
\psi\left(L^{p}, t\right)=t^{1 / p}, \quad \psi\left(L_{h}, t\right)=\frac{1}{h^{-1}\left(t^{-1}\right)}, \quad \psi(\Lambda(\varphi), t)=\psi(M(\varphi), t)=\varphi(t) .
$$


Пространства Лоренща и Марцинкевича играют среди симметричных пространств экстремальную роль: если $\psi$ - фундаментальная функция пространства $X$, то справедлива теорема вложения Е. М. Семенова [5]: $\Lambda(\psi) \subseteq X \subseteq M(\psi)$.

Ниже нам удобно считать, что каждая функция $f: Q \rightarrow \mathbb{R}$ определена не только на $Q$, но и доопределена на все $\mathbb{R}^{n}$ нулем.

Пусть $X$ - симметричное пространство. Для каждой $f: Q \rightarrow \mathbb{R}$ определим модуль непрерывности

$$
\omega(f, h ; X)=\sup _{\|\delta\| \leqslant h}\|f(x+\delta)-f(x) \mid X\|,
$$

где $\|\delta\|=\left|\delta_{1}\right|+\left|\delta_{2}\right|+\cdots+\left|\delta_{n}\right|-$ норма в $\mathbb{R}^{n}$.

Модуль непрерывности $\omega(f, h ; X)$ обладает обычными свойствами:

- $\omega(f, h ; X)$ не убывает по $h$;

$-\omega\left(f, h_{0}+h_{1} ; X\right) \leqslant \omega\left(f, h_{0} ; X\right)+\omega\left(f, h_{1} ; X\right)$;

- для $\alpha>0$ выполнено неравенство $\omega(f, \alpha h ; X) \leqslant(1+\alpha) \omega(f, h ; X)$.

Как обычно, через $\omega(f, h ; X)^{*}$ обозначим наименьшую вогнутую мажоранту функции $\omega(f, h ; X)$. Хорошо известно неравенство С.Б. Стечкина (см., напримep, [6, c. 154])

$$
\omega(f, h ; X) \leqslant \omega(f, h ; X)^{*} \leqslant 2 \omega(f, h ; X) .
$$

Поскольку в дальнейших построениях величина абсолютных констант не играет особой роли, можно считать, что $\omega(f, h ; X)$ - вогнутая функция. Через $\omega\left(f, h ; L^{\infty}\right)$ обозначим равномерный модуль непрерывности.

ОПРеДЕлЕНИЕ 1 . Пусть $X$ - симметричное пространство. Через $H_{X}$ обозначим подпространство в $X$, состоящее из тех $f \in X$, для которых выполнено соотношение

$$
\lim _{h \rightarrow 0} \omega(f, h ; X)=0 .
$$

ЛЕмма 1. Пусть $X$ - правильное симметричное пространство. Тогда $H_{X}=X$.

ДОКАЗАТЕЛЬСТво этой леммы можно провести по стандартной схеме: проверить вьполнение (2) сначала для непрерывной функции, а затем воспользоваться плотностью множества непрерывных функций в $X$; этот факт вытекает из правильности симметричного пространства $X$.

Пусть $\varphi \in U$. Как обьчно, через $H_{X}^{\varphi}$ обозначим пространство функций, норма в котором задается равенством

$$
\left\|f\left|H_{X}^{\varphi}\|=\| f\right| X\right\|+\sup _{h} \frac{\omega(f, h ; X)}{\varphi(h)} .
$$

Задача, рассмотрению которой посвящена настоящая работа, состоит в следующем: пусть $f \in H_{X}^{\varphi}$; нужно найти количественную оценку равномерного модуля непрерывности функции $f$ на "больших" множествах. Для наглядности мы ограничимся случаем $n=2$. При других $n$ рассуждения аналогичны.

Сначала нам потребуются некоторые простые утверждения из теории симметричных пространств. Пусть $X$ - симметричное пространство. Напомним, что оператор $T: X \rightarrow S(m)$ называется квазилинейным, если для всех $f_{0}, f_{1} \in X$, $\alpha, \beta \in \mathbb{R}, \forall x$ выполнено неравенство

$$
\left|T\left\{\alpha f_{0}+\beta f_{1}\right\}(x)\right| \leqslant|\alpha|\left|T\left\{f_{0}\right\}(x)\right|+|\beta|\left|T\left\{f_{1}\right\}(x)\right| .
$$


ЛЕмма 2. Пусть $X$ - симметричное пространство, $\psi(X, t)$ - его фунда-

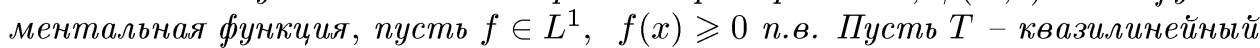
оператор, $T: L^{1} \rightarrow S(\mu)$, и задано $\alpha>0$. Положим $D(T f, \alpha)=\{x:|T f(x)|>$ $\alpha\}$. Если выполнено неравенство

$$
m(D(T f, \alpha)) \leqslant c \alpha^{-1} \int_{D(T f, \alpha)} f(x) d x,
$$

то справедлива оценка

$$
\psi(X, m(D(T f, \alpha))) \leqslant c \alpha^{-1}\|f \mid X\| .
$$

ДоКАЗАТЕЛЬСТво леммы следует из (1) и неравенства

$$
\begin{aligned}
m(D(T f, \alpha)) & \leqslant c \alpha^{-1} \int \chi(D(T f, \alpha)) f(x) d x \leqslant c \alpha^{-1}\left\|f|X\|\| \chi(D(T f, \alpha))| X^{\prime}\right\| \\
& =c \alpha^{-1}\|f \mid X\| \frac{m(D(T f, \alpha))}{\psi(X, m(D(T f, \alpha)))} .
\end{aligned}
$$

Лемма доказана.

Лемма 3. Пусть $X$ - симметричное пространство, $f \in X, f(x) \geqslant 0$ n.в. и задано $\alpha>0$. Положим $D(f, \alpha)=\{x: f(x)>\alpha\}$.

Тогда справедливо неравенство

$$
\psi(X, m(D(f, \alpha))) \leqslant \alpha^{-1}\|f \mid X\| .
$$

ДокАЗАТЕЛЬСтво. Пусть $g \in X^{\prime},\left\|g \mid X^{\prime}\right\| \leqslant 1$ и п.в. вьполнено неравенство $g(x) \geqslant 0$. Тогда, пользуясь свойством Фату, можно написать неравенство

$$
\left\|f|X\|=\| f| X^{\prime \prime}\right\| \geqslant \int g(x) f(x) d x \geqslant \alpha \int \chi(D(f, \alpha)) g(x) d x
$$

и соотношение

$$
\begin{gathered}
\sup \left\{\int \chi(D(f, \alpha)) g(x) d x:\left\|g \mid X^{\prime}\right\| \leqslant 1\right\}=\left\|\chi(D(f, \alpha)) \mid X^{\prime \prime}\right\| \\
=\|\chi(D(f, \alpha)) \mid X\|=\psi(X, m(D(f, \alpha))) .
\end{gathered}
$$

Отсюда и следует (3). Лемма доказана.

Следуюшее построение является ключевым для всей работы.

Зафиксируем $h>0$. Через $B_{h}^{1}$ обозначим множество прямоугольников П в $Q$ вида $\Pi=[a-h, a+h] *[c, d]$, а через $B_{h}^{2}$-множество прямоугольников $\Pi$ в $Q$ вида $\Pi=[c, d] *[a-h, a+h]$. С семействами $B_{h}^{1}$ и $B_{h}^{2}$ свяжем максимальные операторы

$$
M_{h}^{k} f(x)=\sup \left\{\frac{1}{m(\Pi)} \int_{\Pi} f(y) d y: x \in \Pi \in B_{h}^{k}\right\}, \quad k=1,2 .
$$

Оказывается, что семейства $B_{h}^{1}$ и $B_{h}^{2}$ обладают очень хорошими покрываюшими свойствами, что приводит к эффективным оценкам операторов $M_{h}^{k}$. 
Лемма 4. Пусть $\left\{\Pi_{\alpha}: \alpha \in I\right\}$ - семейство прямоугольников из $B_{h}^{1}$. Тогда из семейства $\left\{\Pi_{\alpha}: \alpha \in I\right\}$ можно выбрать последовательность непересекающихся прямоугольников $\left\{\Pi_{i}\right\}$ так, чтобь выполнялось неравенство

$$
m\left(\cup \Pi_{\alpha}: \alpha \in I\right) \leqslant c_{0} \sum m\left(\Pi_{i}\right)
$$

причем константа $c_{0}$ не зависит от $h$. Можно положить $c_{0}=25$.

ДоКАЗАТЕЛЬСТво этой леммы напоминает доказательство известной леммы Витали для шаров (см., например, [7]). Каждому прямоугольнику П = $[a-$ $h, a+h] *[c, d]$ поставим в соответствие число $r(\Pi)=d-c$. Опишем теперь построение последовательности $\Pi_{1}, \Pi_{2}, \ldots, \Pi_{k}, \ldots$ Сначала выберем почти наибольший прямоугольник из семейства $\left\{\Pi_{\alpha}: \alpha \in I\right\}$. Это означает, что $r\left(\Pi_{1}\right)>0,5 \sup \left\{r\left(\Pi_{\alpha}\right): \alpha \in I\right\}$. Предположим, что $\Pi_{1}, \Pi_{2}, \ldots, \Pi_{k}$ уже выбраны. Тогда $\Pi_{k+1}$ выберем так, чтобы он был почти наибольшим прямоугольником среди тех, которые не пересекаются с $\Pi_{1}, \Pi_{2}, \ldots, \Pi_{k}$, т.е.

$$
r\left(\Pi_{k+1}\right)>0,5 \sup \left\{r\left(\Pi_{\alpha}\right): \Pi_{\alpha} \cap\left(\bigcup_{1}^{k} \Pi_{i}\right)=\varnothing, \quad \alpha \in I\right\}
$$

Таким образом получаем последовательность непересекаюшихся прямоугольников $\Pi_{1}, \Pi_{2}, \ldots, \Pi_{k+1}, \ldots$ Из построения семейства $B_{h}^{1}$ следует неравенство

$$
\sum m\left(\Pi_{i}\right) \leqslant m(Q)
$$

Обозначим через $\Pi_{i}^{*}$ прямоугольник, которьй имеет тот же центр, что и $\Pi_{i}$, но раздутый в пять раз. Утверждается, что выполнено соотношение

$$
\left\{\cup \Pi_{\alpha}: \alpha \in I\right\} \subseteq\left\{\cup \Pi_{i}^{*}\right\}
$$

Действительно, зафиксируем $\Pi_{\alpha}$ и выберем первое $k$, обладающее тем свойством, что

$$
r\left(\Pi_{k+1}\right)<0,5 r\left(\Pi_{\alpha}\right)
$$

Тогда $\Pi_{\alpha}$ обязательно пересечет один из прямоугольников $\Pi_{1}, \ldots, \Pi_{k}$. Из простых геометрических соображений следует, что $\Pi_{\alpha}$ содержится в $\left\{\bigcup_{1}^{k} \Pi_{i}^{*}\right\}$. Поэтому из (4) получим неравенство

$$
m\left(\left\{\cup \Pi_{\alpha}: \alpha \in I\right\}\right) \leqslant \sum m\left(\Pi_{i}^{*}\right) \leqslant 25 \sum m\left(\Pi_{i}\right)
$$

Лемма доказана.

С помощью леммы 4 стандартным путем (см., например, [7]) доказывается следующая лемма. 
Лемма 5. Пусть $f \in L^{1} u$ n.в. $f(t) \geqslant 0$. Пусть $\alpha>0$ фиксировано. Положим $D(\alpha, k)=\left\{x: M_{h}^{k} f(x)>\alpha\right\}$.

Тогда справедливо неравенство

$$
m(D(\alpha, k)) \leqslant c_{0} \alpha^{-1} \int_{D(\alpha, k)} f(x) d x
$$

(константа с

Пусть функция $f: Q \rightarrow \mathbb{R}$ имеет частные производные по первой и второй координатам. Введем обозначения

$$
f_{1}(t, s)=\frac{\partial f(t, s)}{\partial t}, \quad f_{2}(t, s)=\frac{\partial f(t, s)}{\partial s} .
$$

Следующая лемма дает хорошие оценки функции $f$ на больших множествах в пространствах Липшица, если известно, что $f_{1} \in X$.

Лемма 6. Пусть $X$ - симметричное пространство. Пусть $f: Q \rightarrow \mathbb{R}$, $f_{1}$ - непрерьвная на $Q$ функиия, причем $f_{1} \in X$. Пусть $\gamma>0$ фиксировано.

Тогда существует множество $U_{1, \gamma}$ такое, что выполнены условия:

а) если $(t, s) \in Q \backslash U_{1, \gamma}$, то для любой точки вида $\left(t_{2}, s\right) \in Q$ верно неравенство

$$
\left|f\left(t_{1}, s\right)-f\left(t_{2}, s\right)\right| \leqslant 2 \gamma\left|t_{1}-t_{2}\right|
$$

б) для меры множества $U_{1, \gamma}$ верно неравенство

$$
\psi\left(X, m\left(U_{1, \gamma}\right)\right) \leqslant c_{0} \gamma^{-1}\left\|f_{1} \mid X\right\|
$$

(константа с с взята из лем.мы 4).

ДоказАТЕЛьство. Сначала по $\gamma$ выберем $h>0$ так, чтобы из неравенства $\left|s_{1}-s_{2}\right|<h$ при всех $t$ выполнялось неравенство

$$
\left|f_{1}\left(t, s_{1}\right)-f_{1}\left(t, s_{2}\right)\right| \leqslant \gamma
$$

Определим теперь множество $U_{1, \gamma}$ равенством

$$
U_{1, \gamma}=\left\{(t, s) \in Q: M_{h}^{2}\left|f_{1}(t, s)\right|>\gamma\right\}
$$

Тогда из лемм 2 и 5 следует неравенство (6).

Пусть теперь $\left(t_{1}, s\right) \in Q \backslash U_{1, \gamma}$ и $\left(t_{2}, s\right) \in Q$. Тогда

$$
\begin{aligned}
& \frac{1}{\left|t_{1}-t_{2}\right|}\left|f\left(t_{1}, s\right)-f\left(t_{2}, s\right)\right|=\frac{1}{\left|t_{1}-t_{2}\right|}\left|\int_{t_{1}}^{t_{2}} f_{1}(\tau, s) d \tau\right| \\
& \leqslant \frac{1}{2 h\left|t_{1}-t_{2}\right|}\left|\int_{-h}^{h} \int_{t_{1}}^{t_{2}} f_{1}(\tau, s+u) d \tau d u\right|+\frac{1}{\left|t_{1}-t_{2}\right|} \\
& \times\left|\int_{t_{1}}^{t_{2}} f_{1}(\tau, s)-\frac{1}{2 h} \int_{-h}^{h} \int_{t_{1}}^{t_{2}} f_{1}(\tau, s+u) d u d \tau\right| \leqslant M_{h}^{2}\left|f_{1}\left(t_{1}, s\right)\right|+\gamma \leqslant 2 \gamma .
\end{aligned}
$$

Тем самым неравенство (5) тоже доказано. Лемма полностью доказана.

Аналогичные факты справедливы и в случае, если известно, что $f_{2}$ непрерывна и $f_{2} \in X$.

Через $Q_{h}(h>0)$ обозначим квадрат вида $Q_{h}=[-h, h] *[-h, h]$, а через $2 d_{h}$ обозначим его диаметр. Для $f \in L^{1}$ положим

$$
f_{h}(x)=\frac{1}{m\left(Q_{h}\right)} \int_{Q_{h}} f(x+y) d y .
$$

Следующие две простые леммы дают оценки средних функций для $f \in X$. В случае $X=L^{p}$ они хорошо известны [8]. 
ЛЕмма 7. Справедливо неравенство

$$
\left\|f_{h_{1}}-f_{h_{2}} \mid X\right\| \leqslant \omega\left(f, d_{h_{1}} ; X\right)+\omega\left(f, d_{h_{2}} ; X\right) .
$$

ДоКАЗАТЕЛЬСтво. Из неравенства

$$
\left|f_{h_{1}}(x)-f_{h_{2}}(x)\right| \leqslant\left|f_{h_{1}}(x)-f(x)\right|+\left|f_{h_{2}}(x)-f(x)\right|
$$

следует, что достаточно найти оценку величины $\left|f_{h}(x)-f(x)\right|$.

Пусть $g \in X^{\prime}$ и $\left\|g \mid X^{\prime}\right\| \leqslant 1$. Тогда можно написать неравенство

$$
\begin{aligned}
\int_{\mathbb{R}^{2}} g(x)\left\{f_{h}(x)-f(x)\right\} d x & =\int_{\mathbb{R}^{2}} g(x) \frac{1}{m\left(Q_{h}\right)} \int_{Q_{h}}\{f(x+y)-f(x)\} d y d x \\
& =\frac{1}{m\left(Q_{h}\right)} \int_{Q_{h}} \int_{\mathbb{R}^{2}} g(x)\{f(x+y)-f(x)\} d y d x \\
& \leqslant \frac{1}{m\left(Q_{h}\right)} \int_{Q_{h}}\left\|g\left|X^{\prime}\|\| f(x+y)-f(x)\right| X\right\| d y \\
& \leqslant \omega\left(f, d_{h} ; X\right) .
\end{aligned}
$$

Переходя к верхней грани по $g$ и вспоминая, что симметричное пространство $X$ обладает свойством Фату, придем к утверждению леммы. Лемма доказана.

ЛЕмма 8. Пусть $X$ - симметричное пространство. При каждом $i=1,2$ верно неравенство

$$
\left\|f_{h, i} \mid X\right\| \leqslant \frac{1}{2 h} \omega(f, 2 h ; X)
$$

(напомним, что $\left.f_{h, 1}(t, s)=\frac{\partial}{\partial t} f_{h}(t, s), f_{h, 2}(t, s)=\frac{\partial}{\partial s} f_{h}(t, s)\right)$.

ДокАЗАТЕЛЬСТВо леммы очевидно.

Теперь мы переходим к основной теореме.

Зафиксируем $\varepsilon>0$, симметричное пространство $X$, функцию $f \in H_{X}$ и модуль непрерывности $\omega(f, h ; X)$, причем мы будем считать, что $\omega(f, h ; X)$ принадлежит $U$, иначе вместо модуля $\omega(f, h ; X)$ нужно взять эквивалентньй модуль $\omega^{*}(f, h ; X)$ и дальше действовать с ним. Выберем теперь две монотонных последовательности положительных чисел $\left\{\delta_{i}\right\}$ и $\left\{\varepsilon_{i}\right\}$ так, чтобы выполнялись условия

$$
\begin{gathered}
\lim _{i \rightarrow \infty} \delta_{i}=0, \\
\sum_{i=1}^{\infty} \varepsilon_{i}<\varepsilon,
\end{gathered}
$$

причем выбор $\left\{\delta_{i}\right\},\left\{\varepsilon_{i}\right\}$ будем осуществлять так, чтобы наряду с (8) и (9) выполнялось соотношение

$$
\sum_{i=1}^{\infty} \frac{\omega\left(f, \delta_{i} ; X\right)}{\psi\left(X ; \varepsilon_{i}\right)}<\infty
$$

Условие (10) означает, что $\omega\left(f, \delta_{i} ; X\right)$ достаточно быстро, а $\psi\left(X ; \varepsilon_{i}\right)$ достаточно медленно стремятся к нулю (напомним, что $\psi(X ; t)$ есть фундаментальная функция пространства $X)$. С последовательностями $\left\{\delta_{i}\right\},\left\{\varepsilon_{i}\right\}$ и модулем непрерывности $\omega(f, h ; X)$ свяжем новую функцию

$$
\Omega(f, h ; X)=\inf _{k}\left\{h \sum_{i=1}^{k} \frac{\omega\left(f, \delta_{i+1} ; X\right)}{\delta_{i+1} \psi\left(X ; \varepsilon_{i}\right)}+\sum_{i=k+1}^{\infty} \frac{\omega\left(f, \delta_{i} ; X\right)}{\psi\left(X ; \varepsilon_{i}\right)}\right\} .
$$

Свойства этой функции описываются в следуюшей лемме. 
ЛЕмма 9. Справедливо соотношение $\Omega(f, h ; X) \in U$.

ДоКАЗАТЕЛЬСТВо леммы следует из определения (11) и соотношения (10).

Теперь у нас все готово для доказательства основной теоремы, но прежде константу $c_{0}$, фигурирующую в (6) и играющую важную роль в теореме 1 , мы зафиксируем.

ТЕОРема 1. Пусть $X$ - симметричное пространство и его фундаментальная функиия $\psi(X ; t)$ строго монотонна. Пусть функиия $f(t, s)$ непрерьвна $u$, следовательно, $f \in H_{X}$. Пусть задано $\varepsilon>0$, и пусть последовательности $\left\{\delta_{i}\right\} \quad u\left\{\varepsilon_{i}\right\}$ удовлетворяют $(8)-(10)$.

Тогда существует множество $W(\varepsilon)$ такое, что выполнены соотношения

$$
m(W(\varepsilon))<\varepsilon
$$

$\operatorname{ecлu}\left(t_{1}, s_{1}\right),\left(t_{2}, s_{2}\right) \in Q \backslash W(\varepsilon)$, mo

$$
\left|f\left(t_{1}, s_{1}\right)-f\left(t_{2}, s_{2}\right)\right| \leqslant\left|f_{\delta_{1}}\left(t_{1}, s_{1}\right)-f_{\delta_{1}}\left(t_{2}, s_{2}\right)\right|+c_{1} \Omega\left(f,\left|t_{1}-t_{2}\right|+\left|s_{1}-s_{2}\right| ; X\right),
$$

где $f_{\delta}-$ средняя функция, а $c_{1}$ - абсолютная константа. Можно положить $c_{1}=8+16 c_{0}$.

ДокАЗАТЕЛЬСтво. Выберем числа $\alpha_{i}$ и $\beta_{i}$ так, чтобы выполнялись соотношения

$$
\begin{aligned}
\frac{\psi\left(X ; \varepsilon_{i}\right)}{2} & =\frac{2}{\alpha_{i}} \omega\left(f, \delta_{i} ; X\right) \\
\frac{\psi\left(X ; \varepsilon_{i}\right)}{2} & =\frac{8 c_{0}}{\beta_{i}} \frac{\omega\left(f, \delta_{i+1} ; X\right)}{\delta_{i+1}}
\end{aligned}
$$

Положим

$$
\begin{aligned}
f(t, s) & =f_{\delta_{1}}(t, s)+\sum_{i=1}^{\infty}\left\{f_{\delta_{i+1}}(t, s)-f_{\delta_{i}}(t, s)\right\} \\
g_{i}(t, s) & =f_{\delta_{i+1}}(t, s)-f_{\delta_{i}}(t, s) \\
g_{i, 1} & =\frac{\partial}{\partial t} g_{i}(t, s), \quad g_{i, 2}=\frac{\partial}{\partial s} g_{i}(t, s) .
\end{aligned}
$$

Функции $g_{i}, g_{i, 1}, g_{i, 2}$ непрерывны, и для них согласно леммам 7 и 8 верны оценки

$$
\begin{gathered}
\left\|g_{i} \mid X\right\| \leqslant \omega\left(f, d_{i} ; X\right)+\omega\left(f, d_{i+1} ; X\right) \leqslant 2 \omega\left(f, d_{i} ; X\right), \\
\left\|g_{i, 1} \mid X\right\| \leqslant \frac{\omega\left(f, 2 \delta_{i+1} ; X\right)}{2 \delta_{i+1}}+\frac{\omega\left(f, 2 \delta_{i} ; X\right)}{2 \delta_{i}} \leqslant \frac{\omega\left(f, 2 \delta_{i+1} ; X\right)}{\delta_{i+1}}, \\
\left\|g_{i, 2} \mid X\right\| \leqslant \frac{\omega\left(f, 2 \delta_{i+1} ; X\right)}{2 \delta_{i+1}}+\frac{\omega\left(f, 2 \delta_{i} ; X\right)}{2 \delta_{i}} \leqslant \frac{\omega\left(f, 2 \delta_{i+1} ; X\right)}{\delta_{i+1}},
\end{gathered}
$$

где $d_{i}=\frac{\sqrt{2}}{2} \delta_{i}$. Из (10) и последних соотношений следует, что ряд, стояший в правой части верхней формулы (16), сходится в $X$ к $f(t, s)$. 
Для каждого $i$ для функции $g_{i}$ применим лемму 6 , полагая $\gamma=\beta_{i} / 2$. Тогда получим множества $U_{i, 1}$ и $U_{i, 2}$, удовлетворяюшие неравенствам

$$
\begin{aligned}
& \psi\left(X ; m\left(U_{i, 1}\right)\right) \leqslant \frac{2 c_{0}\left\|g_{i, 1} \mid X\right\|}{\beta_{i}} \leqslant \frac{2 c_{0}}{\beta_{i}} \frac{\omega\left(f, 2 \delta_{i+1} ; X\right)}{\delta_{i+1}}, \\
& \psi\left(X ; m\left(U_{i, 2}\right)\right) \leqslant \frac{2 c_{0}\left\|g_{i, 2} \mid X\right\|}{\beta_{i}} \leqslant \frac{2 c_{0}}{\beta_{i}} \frac{\omega\left(f, 2 \delta_{i+1} ; X\right)}{\delta_{i+1}},
\end{aligned}
$$

и, следовательно, неравенству

$$
\psi\left(X ; m\left(U_{i, 1} \cup U_{i, 2}\right)\right) \leqslant \frac{4 c_{0}}{\beta_{i}} \frac{\omega\left(f, 2 \delta_{i+1} ; X\right)}{\delta_{i+1}} \leqslant \frac{8 c_{0}}{\beta_{i}} \frac{\omega\left(f, \delta_{i+1} ; X\right)}{\delta_{i+1}} .
$$

Если же $\left(t_{1}, s_{1}\right),\left(t_{2}, s_{2}\right) \in Q \backslash\left(U_{i, 1} \cup U_{i, 2}\right)$, то выполнено неравенство

$$
\begin{aligned}
\left|g_{i}\left(t_{1}, s_{1}\right)-g_{i}\left(t_{2}, s_{2}\right)\right| & \leqslant\left|g_{i}\left(t_{1}, s_{1}\right)-g_{i}\left(t_{1}, s_{2}\right)\right|+\left|g_{i}\left(t_{1}, s_{2}\right)-g_{i}\left(t_{2}, s_{2}\right)\right| \\
& \leqslant \beta_{i}\left(\left|t_{1}-t_{2}\right|+\left|s_{1}-s_{2}\right|\right) .
\end{aligned}
$$

Далее, для функций $g_{i}(t, s)$ введем множества

$$
O_{i}=\left\{(t, s):\left|g_{i}(t, s)\right|>\alpha_{i}\right\}
$$

Тогда из леммы 3 будем иметь

$$
\psi\left(X ; m\left(O_{i}\right)\right) \leqslant \frac{1}{\alpha_{i}}\left\|g_{i} \mid X\right\| \leqslant \frac{2 \omega\left(f, d_{i} ; X\right)}{\alpha_{i}} \leqslant \frac{2 \omega\left(f, \delta_{i} ; X\right)}{\alpha_{i}} .
$$

Положим

$$
W_{i}=U_{i, 1} \cup U_{i, 2} \cup O_{i} .
$$

Тогда из неравенств $(17),(19)$ и соотношений $(14),(15)$ получим

$$
\begin{aligned}
\psi\left(X ; m\left(W_{i}\right)\right) & \leqslant \psi\left(X ; m\left(U_{i, 1} \cup U_{i, 2}\right)\right)+\psi\left(X ; m\left(O_{i}\right)\right) \\
& \leqslant \frac{8 c_{0}}{\beta_{i}} \frac{\omega\left(f, \delta_{i+1} ; X\right)}{\delta_{i+1}}+\frac{2 \omega\left(f, \delta_{i} ; X\right)}{\alpha_{i}} \leqslant \psi\left(X ; \varepsilon_{i}\right) .
\end{aligned}
$$

Из строгой монотонности $\psi(X ; t)$ следует, что

$$
m\left(W_{i}\right) \leqslant \varepsilon_{i}
$$

Положим $W(\varepsilon)=\bigcup_{1}^{\infty} W_{i}$. Тогда из (9) и (20) следует, что выполнено (12).

Если теперь $\left(t_{1}, s_{1}\right),\left(t_{2}, s_{2}\right) \in Q \backslash W(\varepsilon) \subseteq Q \backslash\left(U_{i, 1} \cup U_{i, 2}\right)$, то согласно (18) для любого $i$ выполнено неравенство

$$
\left|g_{i}\left(t_{1}, s_{1}\right)-g_{i}\left(t_{2}, s_{2}\right)\right| \leqslant \beta_{i}\left(\left|t_{1}-t_{2}\right|+\left|s_{1}-s_{2}\right|\right) .
$$

С другой стороны, если $(t, s) \in Q \backslash W(\varepsilon) \subseteq Q \backslash O_{i}$, то для любого $i$ выполнено неравенство

$$
\left|g_{i}(t, s)\right| \leqslant \alpha_{i}
$$


Поэтому для $\left(t_{1}, s_{1}\right),\left(t_{2}, s_{2}\right) \in Q \backslash W(\varepsilon)$ для каждого $k \in \mathbb{N}$ согласно (21) и (22) можно написать неравенство

$$
\begin{aligned}
\left|f\left(t_{1}, s_{1}\right)-f\left(t_{2}, s_{2}\right)\right| \leqslant & \left|f_{\delta_{1}}\left(t_{1}, s_{1}\right)-f_{\delta_{1}}\left(t_{2}, s_{2}\right)\right|+\sum_{i=1}^{\infty}\left|g_{i}\left(t_{1}, s_{1}\right)-g_{i}\left(t_{2}, s_{2}\right)\right| \\
\leqslant & \left|f_{\delta_{1}}\left(t_{1}, s_{1}\right)-f_{\delta_{1}}\left(t_{2}, s_{2}\right)\right|+\sum_{i=1}^{k}\left|g_{i}\left(t_{1}, s_{1}\right)-g_{i}\left(t_{2}, s_{2}\right)\right| \\
& +\sum_{i=k+1}^{\infty}\left|g_{i}\left(t_{1}, s_{1}\right)-g_{i}\left(t_{2}, s_{2}\right)\right| \leqslant\left|f_{\delta_{1}}\left(t_{1}, s_{1}\right)-f_{\delta_{1}}\left(t_{2}, s_{2}\right)\right| \\
& +\left(\left|t_{1}-t_{2}\right|+\left|s_{2}-s_{1}\right|\right) \sum_{i=1}^{k} \beta_{i}+2 \sum_{i=k+1}^{\infty} \alpha_{i} .
\end{aligned}
$$

Из соотношений (14), (15) получим

$$
\begin{aligned}
& \left|f\left(t_{1}, s_{1}\right)-f\left(t_{2}, s_{2}\right)\right| \leqslant\left|f_{\delta_{1}}\left(t_{1}, s_{1}\right)-f_{\delta_{1}}\left(t_{2}, s_{2}\right)\right| \\
& \quad+16 c_{0}\left(\left|t_{1}-t_{2}\right|+\left|s_{2}-s_{1}\right|\right) \sum_{i=1}^{k} \frac{\omega\left(f, \delta_{i+1} ; X\right)}{\delta_{i+1} \psi\left(X ; \varepsilon_{i}\right)}+8 \sum_{i=k+1}^{\infty} \frac{\omega\left(f, \delta_{i} ; X\right)}{\psi\left(X ; \varepsilon_{i}\right)} .
\end{aligned}
$$

Поэтому

$$
\begin{aligned}
& \left|f\left(t_{1}, s_{1}\right)-f\left(t_{2}, s_{2}\right)\right| \leqslant\left|f_{\delta_{1}}\left(t_{1}, s_{1}\right)-f_{\delta_{1}}\left(t_{2}, s_{2}\right)\right| \\
& +c_{1} \inf _{k}\left\{\left(\left|t_{1}-t_{2}\right|+\left|s_{2}-s_{1}\right|\right) \sum_{i=1}^{k} \frac{\omega\left(f, \delta_{i+1} ; X\right)}{\delta_{i+1} \psi\left(X ; \varepsilon_{i}\right)}+\sum_{i=k+1}^{\infty} \frac{\omega\left(f, \delta_{i} ; X\right)}{\psi\left(X ; \varepsilon_{i}\right)}\right\} \\
& \quad \leqslant\left|f_{\delta_{1}}\left(t_{1}, s_{1}\right)-f_{\delta_{1}}\left(t_{2}, s_{2}\right)\right|+c_{1} \Omega(f, h ; X),
\end{aligned}
$$

где $h=\left|t_{1}-t_{2}\right|+\left|s_{1}-s_{2}\right|$, и можно положить $c_{1}=8+16 c_{0}$. Последнее неравенство и доказывает теорему. Теорема доказана.

Обсудим теперь возможности описания "наилучшего" модуля непрерывности $\Omega(f, h ; X)$ в (13), причем желательно "минимизировать" $\Omega(f, h ; X)$ по последовательностям $\left\{\varepsilon_{i}\right\}$ и $\left\{\delta_{i}\right\}$. Если никакой дополнительной информации нет, то применим подход К. Осколкова [1]-[3], который заключается в следуюшем.

Пусть $\omega(f, h ; X)$ удовлетворяет условию

$$
\lim _{h \rightarrow 0} \frac{\omega(f, h ; X)}{h}=\infty
$$

Положим $\delta_{0}=0,5$, и пусть точки $\delta_{1}, \ldots, \delta_{n}$ определены. Определим теперь точку $\delta_{n+1}$ равенством

$$
\delta_{n+1}=\sup \left\{\delta: \frac{\omega(f, \delta ; X)}{\delta} \geqslant 2 \frac{\omega\left(f, \delta_{n} ; X\right)}{\delta_{n}} \text { и } \omega(f, \delta ; X) \leqslant 0,5 \omega\left(f, \delta_{n} ; X\right)\right\} .
$$

Тогда для последовательности $\left\{\varepsilon_{i}\right\}$, удовлетворяющей (9) и дополнительному соотношению

$$
\lim _{i \rightarrow \infty} \frac{\varepsilon_{i+1}}{\varepsilon_{i}}=1
$$


модуль $\Omega(f, h ; X)$ допускает для $h \in\left[\delta_{k}, \delta_{k-1}\right]$ оценку

$$
\Omega(f, h ; X) \leqslant \bar{c} \frac{\Omega(f, h ; X)}{\psi\left(X ; \varepsilon_{k}\right)},
$$

где $\bar{c}$ зависит только от $\left\{\varepsilon_{i}\right\}$.

Действительно, из (24) следует, что выполнены неравенства

$$
\frac{\omega\left(f, \delta_{n+1} ; X\right)}{\delta_{n+1}} \geqslant 2 \frac{\omega\left(f, \delta_{n} ; X\right)}{\delta_{n}}, \quad \omega\left(f, \delta_{n+1} ; X\right) \leqslant 0,5 \omega\left(f, \delta_{n} ; X\right) .
$$

Поэтому из $(25)$ и $(27)$ следует, что найдется константа $c$ такая, что

$$
\begin{gathered}
\sum_{i=1}^{k-1} \frac{\omega\left(f, \delta_{i+1} ; X\right)}{\delta_{i+1} \psi\left(X ; \varepsilon_{i}\right)} \leqslant c \frac{\omega\left(f, \delta_{k} ; X\right)}{\delta_{k} \psi\left(X ; \varepsilon_{k-1}\right)} \leqslant c \frac{\omega\left(f, \delta_{k} ; X\right)}{\delta_{k} \psi\left(X ; \varepsilon_{k}\right)} \\
\sum_{i=k}^{\infty} \frac{\omega\left(f, \delta_{i} ; X\right)}{\psi\left(X ; \varepsilon_{i}\right)} \leqslant c \frac{\omega\left(f, \delta_{k} ; X\right)}{\psi\left(X ; \varepsilon_{k}\right)} .
\end{gathered}
$$

Отсюда следует, что при $h \in\left[\delta_{k}, \delta_{k-1}\right]$ для $\Omega(f, h ; X)$ можно написать оценку

$$
\Omega(f, h ; X) \leqslant c \min \left\{\frac{\omega\left(f, \delta_{k} ; X\right)}{\psi\left(X ; \varepsilon_{k}\right)}\left(\frac{h}{\delta_{k}}+1\right), \frac{\omega\left(f, \delta_{k-1} ; X\right)}{\psi\left(X ; \varepsilon_{k-1}\right)}\left(\frac{h}{\delta_{k-1}}+1\right)\right\} .
$$

Если выполнено равенство

$$
\omega\left(f, \delta_{k} ; X\right)=0,5 \omega\left(f, \delta_{k-1} ; X\right),
$$

TO

$$
\begin{aligned}
\Omega(f, h ; X) & \leqslant c \frac{\omega\left(f, \delta_{k-1} ; X\right)}{\psi\left(X ; \varepsilon_{k-1}\right)}\left(\frac{h}{\delta_{k-1}}+1\right) \\
& \leqslant 2 c \frac{\omega\left(f, \delta_{k-1} ; X\right)}{\psi\left(X ; \varepsilon_{k-1}\right)} \leqslant 4 c \frac{\omega\left(f, \delta_{k} ; X\right)}{\psi\left(X ; \varepsilon_{k-1}\right)} \leqslant 4 c \frac{\omega(f, h ; X)}{\psi\left(X ; \varepsilon_{k}\right)} .
\end{aligned}
$$

Если же выполнено другое равенство -

$$
\frac{\omega\left(f, \delta_{k} ; X\right)}{\delta_{k}}=2 \frac{\omega\left(f, \delta_{k-1} ; X\right)}{\delta_{k-1}},
$$

то

и поэтому

$$
\frac{\omega\left(f, \delta_{k} ; X\right)}{\delta_{k}} \leqslant \frac{\omega(f, h ; X)}{h},
$$

$$
\Omega(f, h ; X) \leqslant c \frac{\omega\left(f, \delta_{k} ; X\right)}{\psi\left(X ; \varepsilon_{k}\right)}\left(\frac{h}{\delta_{k}}+1\right) \leqslant 2 c \frac{\omega(f, h ; X)}{\psi\left(X ; \varepsilon_{k}\right)} .
$$

Таким образом, оценка (26) верна.

Ниже последовательность $\left\{\delta_{k}\right\}$, определенную равенством (24), будем называть $K$-последовательностью для модуля непрерывности $\omega(f, \delta ; X)$. Полезно отметить, что $K$-последовательность $\left\{\delta_{k}\right\}$ для модуля непрерывности $\omega(f, \delta ; X)$ убывает не медленнее геометрической прогрессии и $\lim _{k \rightarrow \infty} \delta_{k}=0$.

С использованием $K$-последовательности теорему 1 можно переформулировать в следующем виде. 
Теорема 2. Пусть $X$ - симметричное пространство, причем его фундаментальная функиия $\psi(X ; t)$ строго монотонна. Пусть $f(t, s)$ непрерьвна $u$, следовательно, $f \in H_{X}$. Пусть модуль непрерьвности $\omega(f, \delta ; X)$ удовлетворяет условию (23) и $\left\{\delta_{k}\right\}$ есть $K$-последовательность для $\omega(f, \delta ; X)$. Пусть задано $\varepsilon>0$, а последовательность $\left\{\varepsilon_{i}\right\}$ удовлетворяет условиям (9) и (25).

Тогда существует множество $W(\varepsilon)$ такое, что выполнены соотношения

$$
\begin{gathered}
m(W(\varepsilon))<\varepsilon ; \\
\text { если }\left(t_{1}, s_{1}\right),\left(t_{2}, s_{2}\right) \in Q \backslash W(\varepsilon) \quad u \quad h=\left|t_{1}-t_{2}\right|+\left|s_{1}-s_{2}\right| \in\left[\delta_{k}, \delta_{k-1}\right], m o \\
\left|f\left(t_{1}, s_{1}\right)-f\left(t_{2}, s_{2}\right)\right| \leqslant C\left\{\left|f_{\delta_{1}}\left(t_{1}, s_{1}\right)-f_{\delta_{1}}\left(t_{2}, s_{2}\right)\right|+\frac{\omega(f, h ; X)}{\psi\left(X ; \varepsilon_{k}\right)}\right\},
\end{gathered}
$$

где $C$ - константа, зависящая только от $\left\{\varepsilon_{i}\right\}$.

Теорема 2 показывает, что все пространства $H_{X}^{\varphi}$, построенные по симметричному пространству $X$ с данной фундаментальной функцией, на "больших" множествах допускают одинаково ухудшаюшую оценку равномерного модуля непрерывности, т.е. $\omega\left(f, h ; L^{\infty}\right)$ увеличивается по сравнению $\mathrm{c} \omega(f, h ; X)$ на один и тот же множитель. В этом смысле оценки равномерного модуля непрерывности у функций из $H_{X}^{\varphi}$ зависят лишь от фундаментальной функции симметричного пространства $X$.

Сейчас мы покажем неусиляемость теоремы 2. Все построения будут проводиться на $\mathbb{R}$, причем основной контрпример будет дан для периодического случая. Поэтому ниже мы будем рассматривать периодические функции с периодом 1 и соответствуюшие симметричные пространства.

Нам потребуются некоторые предварительные построения.

Положим $\theta_{0}(t)=1-2|t-0,5|$ для $t \in[0,1]$ и продолжим ее на $\mathbb{R}$ периодически с периодом 1. Зафиксируем $\psi \in U$ и три числа: $0,5>b_{k}>0, a_{k}>0, b_{k}>\tau_{k}>0$.

Пусть $\left[\frac{b_{k}}{\tau_{k}}\right]$ есть целая часть числа $\frac{b_{k}}{\tau_{k}}$. Определим функцию

$$
\theta_{k}(t)= \begin{cases}a_{k} \theta_{0}\left(\frac{t}{\tau_{k}}\right) & \text { при } t \in\left[0, \tau_{k}\left[\frac{b_{k}}{\tau_{k}}\right]\right) \\ 0 & \text { при } t \in\left[\tau_{k}\left[\frac{b_{k}}{\tau_{k}}\right], 1\right)\end{cases}
$$

и продолжим на $\mathbb{R} \backslash[0,1]$ по периодичности. Нетрудно видеть, что график $\theta_{k}(t)$ есть "пила". Нам потребуются оценки нормы функции $\theta_{k}(t)$ в пространствах Лоренца $\Lambda(\psi)$ и Марцинкевича $M(\psi)$.

Лемма 10. Справедливо неравенство

$$
\left\|\theta_{k}(t+h)-\theta_{k}(t) \mid \Lambda(\psi)\right\| \leqslant 4 a_{k} \psi\left(b_{k}\right) \min \left\{\frac{h}{\tau_{k}}, 1\right\} .
$$


ДоказАТЕЛЬСтво. Обозначим через $\lambda_{k}(\alpha, h)$ функцию распределения функции $\theta_{k}(t+h)-\theta_{k}(t)$ :

$$
\lambda_{k}(\alpha, h)=m\left(\left\{t \in[0,1]:\left|\theta_{k}(t+h)-\theta_{k}(t)\right|>\alpha\right\}\right) .
$$

Сразу же отметим, что при всех $\alpha>0$ выполнено неравенство

$$
\lambda_{k}(\alpha, h) \leqslant 2 b_{k}
$$

апри $\alpha>a_{k}$ верно соотношение

$$
\lambda_{k}(\alpha, h)=0 .
$$

Поэтому если $h>0,5 \tau_{k}$, то из $(29),(30)$ получим

$$
\begin{aligned}
\left\|\theta_{k}(t+h)-\theta_{k}(t) \mid \Lambda(\psi)\right\| & =\int_{0}^{\infty} \psi\left(\lambda_{k}(\alpha, h)\right) d \alpha \\
=\int_{0}^{a_{k}} \psi\left(\lambda_{k}(\alpha, h)\right) d \alpha & \leqslant a_{k} \psi\left(2 b_{k}\right) \leqslant 2 a_{k} \psi\left(b_{k}\right) .
\end{aligned}
$$

Если же $h<0,5 \tau_{k}$, то сначала выберем $i_{0}$ так, чтобы

$$
a_{k} 2^{-\left(i_{0}+1\right)} \leqslant \theta_{k}(h)<a_{k} 2^{-i_{0}} .
$$

Тогда при $\alpha>a_{k} 2^{-i_{0}}$ будем иметь $\lambda_{k}(\alpha, h)=0$. Поэтому

$$
\begin{aligned}
& \left\|\theta_{k}(t+h)-\theta_{k}(t) \mid \Lambda(\psi)\right\|=\int_{0}^{\infty} \psi\left(\lambda_{k}(\alpha, h)\right) d \alpha \\
& \quad=\int_{0}^{a_{k} 2^{-i_{0}}} \psi\left(\lambda_{k}(\alpha, h)\right) d \alpha \leqslant a_{k} 2^{-i_{0}} \psi\left(2 b_{k}\right) \leqslant 4 \psi\left(b_{k}\right) \theta_{k}(h) .
\end{aligned}
$$

Объединяя (31) и (32), придем к (28). Лемма доказана.

ЛЕмма 11. При $h \in\left[0,0,25 \tau_{k}\right]$ справедливо неравенство

$$
\left\|\theta_{k}(t+h)-\theta_{k}(t) \mid \Lambda(\psi)\right\| \geqslant 0,25 a_{k} \psi\left(b_{k}\right) \frac{h}{\tau_{k}} .
$$

ДоКАЗАТЕЛЬСТВо. Положим

$$
D_{h}=\left\{t \in\left[0, b_{k}\right]:\left|\theta_{k}(t+h)-\theta_{k}(t)\right|>\left|\theta_{k}(h)\right|\right\} .
$$

Тогда из графика $\theta_{k}(t)$ легко видеть, что выполнено неравенство $m\left(D_{h}\right) \geqslant$ $0,25 b_{k}$. Поэтому

$$
\begin{aligned}
\left\|\theta_{k}(t+h)-\theta_{k}(t) \mid M(\psi)\right\| & \geqslant \frac{\psi\left(m\left(D_{h}\right)\right)}{m\left(D_{h}\right)} \int_{D_{h}}\left|\theta_{k}(t+h)-\theta_{k}(t)\right| d t \\
& \geqslant \theta_{k}(h) \psi\left(0,25 b_{k}\right) \geqslant 0,25 \theta_{k}(h) \psi\left(b_{k}\right) .
\end{aligned}
$$

Из последнего неравенства легко следует (33). Лемма доказана.

Из лемм 10 и 11 и теоремы вложения Е. М. Семенова [5] получим следующую теорему. 
ТЕОРема 3. Пусть $X$ - симметричное пространство, $\psi \in U-$ его фундаментальная функиия.

Тогда существует константа с, зависящая только от X и такая, что при $h \in[0,0,5]$ выполнено неравенство

$$
c^{-1} a_{k} \psi\left(b_{k}\right) \min \left\{\frac{h}{\tau_{k}}, 1\right\} \leqslant \omega\left(\theta_{k}, h ; X\right) \leqslant c a_{k} \psi\left(b_{k}\right) \min \left\{\frac{h}{\tau_{k}}, 1\right\} .
$$

Теперь мы сформулируем теорему, показывающую неусиляемость теоремы 2.

ТЕОРема 4. Пусть $X$ - симметричное пространство с фундаментальной функцией $\psi \in U$. Пусть $v(t)$ - модуль непрерьвности, для которого вьполнено (23). Пусть $\left\{\delta_{i}\right\}$ есть $K$-последовательность для $v(t)$. Пусть для последовательности $\left\{b_{i}\right\}$ выполнень соотношения

$$
0,5 \geqslant b_{i} \geqslant b_{i+1}, \quad \lim _{i \rightarrow \infty} \frac{b_{i}}{b_{i+1}}=1, \quad \sum_{i=1}^{\infty} b_{i}=\infty
$$

Тогда найдется $f \in H_{X}^{v}$ такая, что для любого множсества $M \quad c$ $m(M)>0$ выполнено соотношение

$$
\varlimsup_{k \rightarrow \infty} \frac{\psi\left(b_{k}\right) \sup \left\{|f(t)-f(s)|: t, s \in M,|t-s| \leqslant \delta_{k}\right\}}{v\left(\delta_{k}\right)}>0 .
$$

ДокаЗАтельство. Так как $\left\{\delta_{k}\right\}$ есть $K$-последовательность, то при $h \in[0,1]$ вьполнено неравенство

$$
\sum_{k=1}^{\infty} v\left(\delta_{k}\right) \min \left\{\frac{h}{\delta_{k}}, 1\right\} \leqslant v(h)
$$

Доказательство (36) можно найти в [3] или получить так же, как доказательство (26).

Положим $a_{k}=\frac{v\left(\delta_{k}\right)}{\psi\left(b_{k}\right)}$. Из того, что $\left\{\delta_{k}\right\}$ есть $K$-последовательность, и из $(34)$ следует, что при больших $i$ выполнены неравенства

$$
\begin{aligned}
a_{i+1} \leqslant & \frac{3}{4} a_{i}, \quad \frac{a_{i+1}}{\delta_{i+1}} \geqslant \frac{4}{3} \frac{a_{i}}{\delta_{i}}, \\
& \lim _{i \rightarrow \infty} \frac{b_{i}}{\delta_{i}}=\infty .
\end{aligned}
$$

Поэтому мы будем считать, что при всех $i b_{i}>\delta_{i}$. По последовательностям чисел $\left\{a_{k}, \delta_{k}, b_{k}\right\}$ построим функции $\theta_{k}$.

Определим новую последовательность чисел $d_{k} \rightarrow \infty$ равенствами

$$
d_{1}=0, \quad \ldots, \quad d_{k}=\sum_{i=1}^{k-1} b_{i}, \quad \ldots, \quad k=2,3, \ldots
$$


а последовательность функций соотношениями

$$
\begin{array}{ll}
g_{i}(t)=\left\{\begin{array}{lll}
\theta_{i}\left(t-d_{i}\right) & \text { при } t \in\left(d_{i}, d_{i+1}\right], \\
0 & \text { при } t \in R \backslash\left(d_{i}, d_{i+1}\right],
\end{array}\right. \\
f_{i}(t)=\sum_{i=0}^{\infty} g_{i}(t+k), & f(t)=\sum_{i=1}^{\infty} f_{i}(t+k) .
\end{array}
$$

Функции $f_{i}$ и $f$ периодические с периодом 1 . Из построения функций $f_{i}$ следует, что при $0 \leqslant h<0,25$ выполнены соотношения

$$
\omega(f, h ; X)=\omega\left(\theta_{i}, h ; X\right), \quad\left\|f_{i}\left|X\|\leqslant c\| f_{i}\right| \Lambda(\psi)\right\| \leqslant c a_{i} \psi\left(b_{i}\right) .
$$

Поэтому из теоремы 3 и соотношений $(34),(36)$ и (37) следует, что $f \in X$ и

$$
\omega(f, h ; X)=\omega\left(\sum_{i=1}^{\infty} f_{i}, h ; X\right) \leqslant \sum_{i=1}^{\infty} \omega\left(f_{i}, h ; X\right) \leqslant c \sum_{i=1}^{\infty} v\left(\delta_{i}\right) \min \left\{\frac{h}{\delta_{i}}, 1\right\} \leqslant c_{1} v(h)
$$

Таким образом, $f \in H_{X}^{v}$.

Покажем теперь, что вьполнено (35). Для этого проведем некоторые вспомогательные построения.

Для каждого $t \in[0,1)$ и $i \in \mathbb{N}$ положим $p(t, i)=\inf \left\{k: t+i \leqslant \sum_{j=1}^{k} b_{j}=d_{k+1}\right\}$ (напомним, что выполнено $(34)$, и поэтому $p(t, i)<\infty)$. Так как $\sum_{j=1}^{p(t, i)-1} b_{j}<$ $t+i \leqslant \sum_{j=1}^{p(t, i)} b_{j}$, то $1 \leqslant \sum_{j=p(t, i)}^{p(t, i+1)} b_{j}$. Поэтому из монотонности $b_{j}$ получим

$$
p(t, i+1)-p(t, i) \geqslant \frac{1}{b_{p(t, i)}}
$$

и, следовательно,

$$
\lim _{i \rightarrow \infty}\{p(t, i+1)-p(t, i)\}=\infty .
$$

Так же доказывается, что

$$
\lim _{i \rightarrow \infty} \inf _{t \in[0,1)}\{p(t, i+1)-p(t, i)\}=\infty
$$

Из соотношений (37) и (39) следует

ЛЕмма 12. Существует номер $i_{0}$ такой, что если $i \geqslant i_{0}$, то для любого $t \in[0,1)$ справедливы соотношения

$$
\sum_{j=p(t, i+1)}^{\infty} a_{j}<\frac{1}{16} a_{p(t, i)}, \quad \sum_{j=1}^{p(t, i-1)} \frac{a_{j}}{\delta_{j}}<\frac{1}{16} \frac{a_{p(t, i)}}{\delta_{p(t, i)}}
$$

Кроме того, нам потребуется следующая лемма из метрической теории функций. 
Лемма 13. Пусть мера множества $M$ положительна. Пусть $t-$ точка плотности множества $M$.

Тогда существует $\delta(t)$ такое, что если $0<\delta \leqslant \delta(t)$, то найдется $h \in$ $[0,5 \delta, 0,75 \delta]$, для которого точки $\tau_{1}=t-2 h, \tau_{2}=t-h, \tau_{3}=t, \tau_{4}=t+h$, $\tau_{5}=t+2 h$ принадлежат множеству $M$.

Теперь у нас все готово для проверки свойства (35).

Пусть $t$ - точка плотности множества $M$. Согласно лемме 13 найдем число $\delta(t)$. Выберем теперь $n(\delta) \in \mathbb{N}$ так, чтобы при $k>n(\delta)$ выполнялось неравенство $\delta_{k}<\delta(t)$. Для каждого $i \in \mathbb{N}$ определим $k(i) \in \mathbb{N}$ так, чтобы $t+i \in\left(d_{k(i)}, d_{k(i)+1}\right]$ (напомним, что $d_{l}=\sum_{j=1}^{l-1} b_{j}$ ). Выберем теперь $i$ и соответственно $k(i)$ так, чтобы выполнялись следующие требования:

$$
\begin{aligned}
i & >i_{0} \quad\left(i_{0} \quad \text { из леммы } 12\right) ; \\
k(i) & >n(\delta) \quad(n(\delta) \quad \text { определено вьше); } \\
b_{k(i)} & >2 \delta_{k(i)} .
\end{aligned}
$$

Последнее неравенство возможно в силу (38). Применим теперь лемму 13 для $\delta=$ $0,5 \delta_{k(i)}$ (в силу $(40) k(i)>k(\delta)$, и поэтому $0,5 \delta_{k(i)}<\delta(t)$ ). Получим точки $\tau_{1}, \tau_{2}, \tau_{3}, \tau_{4}, \tau_{5}$. Выберем среди этих точек две соседние так, чтобы они были расположены на одном участке линейности функции $f_{k(i)}$, а сдвинутые на $i$ лежали бы в $\left(d_{k(i)}, d_{k(i)+1}\right]$. Такой выбор всегда возможен. Для определенности мы будем считать, что это точки $t$ и $t+h$ и соответственно

$$
\left|f_{k(i)}(t+h)-f_{k(i)}(t)\right|=2 h \frac{a_{k(i)}}{\delta_{k(i)}} .
$$

Оценим теперь прирашение функции $f$ :

$$
\begin{aligned}
& |f(t+h)-f(t)|=\left|\sum_{j=1}^{\infty}\left(f_{j}(t+h)-f_{j}(t)\right)\right| \\
& \geqslant\left|f_{k(i)}(t+h)-f_{k(i)}(t)\right|-\sum_{j<k(i)}\left|\left(f_{j}(t+h)-f_{j}(t)\right)\right| \\
& \quad+\sum_{j>k(i)}\left|\left(f_{j}(t+h)-f_{j}(t)\right)\right| \geqslant 2 h \frac{a_{k(i)}}{\delta_{k(i)}}-A_{k(i)}-B_{k(i)} .
\end{aligned}
$$

Так как в суммах для $A_{k(i)}$ и $B_{k(i)}$ большинство слагаемых равны нулю, то с использованием индексов $p(t, j)$ можно написать оценки

$$
\begin{aligned}
B_{k(i)} & \leqslant \sum_{j>k(i)} f_{j}(t+h)+\sum_{j>k(i)} f_{j}(t) \leqslant \sum_{j=p(t, i+1)}^{\infty} a_{j}+\sum_{j=p(t+h, i+1)}^{\infty} a_{j}, \\
A_{k(i)} & \leqslant \sum_{j \leqslant p(t, i-1)}^{\infty}\left|f_{j}(t+h)-f_{j}(t)\right|+\sum_{j \leqslant p(t+h, i-1)}^{\infty}\left|f_{j}(t+h)-f_{j}(t)\right| \\
& \leqslant 2 h \sum_{j \leqslant p(t, i-1)}^{\infty} \frac{a_{j}}{\delta_{j}}+2 h \sum_{j \leqslant p(t+h, i-1)}^{\infty} \frac{a_{j}}{\delta_{j}},
\end{aligned}
$$


Согласно (39) $i>i_{0}$, поэтому из леммы 12 следует, что

$$
\begin{aligned}
\sum_{j=p(t, i+1)}^{\infty} a_{j}<\frac{1}{16} a_{p(t, i)}, & \sum_{j=p(t+h, i+1)}^{\infty} a_{j}<\frac{1}{16} a_{p(t+h, i)}, \\
\sum_{j=p(t, i-1)}^{\infty} \frac{a_{j}}{\delta_{j}}<\frac{1}{16} \frac{a_{p(t, i)}}{\delta_{p(t, i)}}, & \sum_{j=p(t+h, i-1)}^{\infty} \frac{a_{j}}{\delta_{j}}<\frac{1}{16} \frac{a_{p(t+h, i)}}{\delta_{p(t+h, i)}} .
\end{aligned}
$$

В силу выбора $t+i, t+i+h \in\left(d_{k(i)}, d_{k(i)+1}\right]$ и, следовательно, $p(t, i)=p(t+h, i)=$ $k(i)$. Поэтому

$$
B_{k(i)}<\frac{1}{8} a_{k(i)}, \quad A_{k(i)}<\frac{h}{4} \frac{a_{k(i)}}{\delta_{k(i)}}
$$

Следовательно,

$$
|f(t+h)-f(t)|>\frac{7}{4} \frac{a_{k(i)}}{\delta_{k(i)}} h-\frac{1}{8} a_{k(i)} .
$$

Так как $h \in\left[0,25 \delta_{k(i)}, 0,375 \delta_{k(i)}\right]$, то из (40) следует (34). Теорема полностью доказана.

Проиллюстрируем приведенные теоремы примером. Пусть $v(t)=t^{\alpha} \quad(0<$ $\alpha<1)$. Этот модуль непрерывности степенной. Для него $K$-последовательность легко считается и представляет собой геометрическую прогрессию. Пусть $f \in$ $H_{X}^{v}$. Полагая $\varepsilon_{i}=c \varepsilon i^{-(1+\gamma)}(\gamma>0)$, из теорем 1,2 получим, что найдется множество меры больше $1-\varepsilon$, на котором равномерньй модуль непрерывности допускает оценку

$$
\omega\left(f, h ; L^{\infty}\right) \leqslant c_{1} \frac{h^{\alpha}}{\psi\left(X ;\left(\ln h^{-1}\right)^{-(1+\gamma)}\right)} ;
$$

если же положить $\varepsilon_{i}=c \varepsilon i^{-1}(\ln i)^{-(1+\gamma)}$, то найдется множество меры больше $1-\varepsilon$, на котором равномерньй модуль непрерывности допускает оценку

$$
\omega\left(f, h ; L^{\infty}\right) \leqslant c_{2} \frac{h^{\alpha}}{\psi\left(X ;\left(\ln h^{-1}\right)^{-1}\left(\ln \ln h^{-1}\right)^{-(1+\gamma)}\right)},
$$

и т.д. С другой стороны, полагая $b_{i}=i^{-1}$, из теоремы 4 получаем, что найдется $f \in H_{X}^{v}$, для которой равномерный модуль непрерьвности, вычисленный на любом множестве положительной меры, допускает оценку

$$
\varlimsup_{h \rightarrow 0} \frac{\omega\left(f, h ; L^{\infty}\right) \psi\left(X ;\left(\ln h^{-1}\right)^{-1}\right)}{h^{\alpha}}>0 ;
$$

если положить $b_{i}=(i \ln i)^{-1}$ и применить теорему 4 , то найдется $f_{1} \in H_{X}^{v}$, для которой равномерный модуль непрерывности, вычисленный на любом множестве положительной меры, допускает оценку

$$
\varlimsup_{h \rightarrow 0} \frac{\omega\left(f_{1}, h ; L^{\infty}\right) \psi\left(X ;\left(\ln \ln h^{-1}\right)^{-1}\left(\ln h^{-1}\right)^{-1}\right)}{h^{\alpha}}>0 .
$$

Таким образом, "наилучшей” оценки модуля непрерывности в рассматриваемой ситуации нет. 
ЗАмЕчАниЕ 1. При доказательстве теорем 1,2 предполагалось, что $f \in H_{X}$ непрерывна. Отметим, что единственное место, где использовалась непрерывность, это лемма 6 . Интересно было бы узнать, каковы минимальные требования на $f \in H_{X}$ для справедливости теорем 1,2 при $n>1$, так как при $n=1$ требование непрерьвности для $f \in H_{X}$ легко снимается. Здесь также уместно заметить, что небольшой модификацией функций $\theta_{k}$ в теореме 4 можно добиться, чтобы построенная там функция $f$ была непрерывна.

ЗАмЕчАниЕ 2. При доказательстве теорем 1,2 предполагалось, что фундаментальная функция $\psi(X ; t)$ симметричного пространства $X$ является строго монотонной. Это свойство использовалось лишь при доказательстве неравенства (20). Сейчас мы покажем, что если

$$
\lim _{t \rightarrow 0} \psi(X ; t)=0
$$

то на этом симметричном пространстве $X$ найдется эквивалентная первоначальной симметричная норма $\|f \mid X\|_{1}$ такая, что новая фундаментальная функция $\psi_{1}(X ; t)=\|\chi(D) \mid X\|(m(D)=t)$ будет строго монотонной. Проверить этот факт можно следующим образом. Для функции $\psi(X ; t)$ найдем эквивалентную ей строго монотонную вогнутую функцию $\varphi_{0}(t)$ :

$$
\varphi_{0}(t) \leqslant \psi(X ; t) \leqslant c \varphi_{0}(t), \quad t \in[0,1],
$$

и по $\varphi_{0}$ построим пространство Лоренца $\Lambda\left(\varphi_{0}\right)$. Введем новое симметричное пространство $\left(\Lambda\left(\varphi_{0}\right)+X\right)_{d}$. Оно состоит из функций, для которых конечна норма

$$
\begin{aligned}
\left\|f \mid\left(\Lambda\left(\varphi_{0}\right)+X\right)_{d}\right\| & =\inf \left\{\left\|f_{0}\left|\Lambda\left(\varphi_{0}\right)\|+\| f_{1}\right| X\right\|:\right. \\
f & \left.=f_{0}+f_{1}, \operatorname{supp} f_{0} \cap \operatorname{supp} f_{0}=\varnothing\right\} .
\end{aligned}
$$

Тогда из теоремы вложения Е. М. Семенова и из (42) следует с точностью до эквивалентных норм равенство $\left(\Lambda\left(\varphi_{0}\right)+X\right)_{d}=X$. С другой стороны, из (42) следует, что при $t \in[0,1]$ верно равенство

$$
\begin{gathered}
\psi\left(\left(\Lambda\left(\varphi_{0}\right)+X\right)_{d} ; t\right)=\varphi_{0}(t) . \\
\text { Далее достаточно положить }\left\|f\left|X\left\|_{1}=\right\| f\right|\left(\Lambda\left(\varphi_{0}\right)+X\right)_{d}\right\| .
\end{gathered}
$$

\section{Список литературы}

1. Осколков K.И. Аппроксимативные свойства суммируемых функций на множествах полной меры // Матем. сб. 1977. Т. 103. № 4. С. 563-589.

2. Осколков К.И. Равномерный модуль непрерывности суммируемых функций на множествах положительной меры // ДАН СССР. 1976. Т. 229. С. 304-306.

3. Ульянов П. Л. О работах Н.Н. Лузина по метрической теории функций // УМН. 1985. T. 40. № 3. C. $15-70$.

4. Брудный Ю.А. Пространства, определяемые с помощью локальных приближений // Tp. MMO. 1971. T. 24. C. 69-132.

5. Крейн С. Г., Петунин Ю. И., Семенов Е. М. Интерполяция линейных операторов. М.: Наука, 1977.

6. Дзядык B. К. Введение в теорию равномерного приближения функций полиномами. М.: Наука, 1977.

7. Стейн И. Сингулярные интегралы и дифференциальные свойства функций. М.: Мир, 1973.

8. Тиман A. Ф. Теория приближения функций действительного переменного. М.: Гостехиздат, 1960.

Ярославский государственный университет 